\title{
Validez y confiabilidad del cuestionario Florida versión en español
}

\section{Diana M. Castillo-Sierra ${ }^{a, *}$, Renata V. González-Consuegra ${ }^{a}$ y Alejandro Olaya-Sánchez ${ }^{a, b}$}

a Facultad de Enfermería, Universidad Nacional de Colombia, Bogotá, Colombia

b Hospital Cardiovascular del Niño de Cundinamarca, Bogotá, Colombia

Recibido el 10 de septiembre de 2017; aceptado el 20 de diciembre de 2017

Disponible en Internet el 7 de marzo de 2018

\section{PALABRAS CLAVE \\ Estimulación \\ eléctrica; \\ Dispositivo; \\ Estudios de \\ validación; \\ Aceptación de la atención en salud; \\ Arritmias}

\begin{abstract}
Resumen
Antecedentes: Para los sistemas de salud actuales, la medición y la generación de indicadores que permitan evaluar los resultados en salud, han tomado gran relevancia como parte de la calidad en la atención. En consecuencia, los procesos de adaptación y validación de los instrumentos, son importantes para tener herramientas de medición fiables. El instrumento Florida Patient Acceptance Survey, es una escala específica para medir la aceptación de las personas a los dispositivos cardiacos.

Método: estudio de tipo metodológico, en el cual se llevó a cabo la validez facial, de contenido y confiabilidad de la versión en español del instrumento Florida Patient Acceptance Survey. La validez facial y de contenido se realizó mediante el juicio de expertos, utilizando las pruebas estadísticas de Coeficiente Kappa de Fleiss e índice de Lawshe modificado. Para realizar la prueba de confiabilidad se utilizó la teoría del ítem; participaron 126 personas implante de un dispositivo de estimulación cardiaca. La fiabilidad se calculó mediante la prueba estadística de alfa de Cronbach.

Resultados: la validez facial evaluó tres categorías: claridad, precisión y comprensión, mostrando un acuerdo moderado entre los jueces para las categorías. La validez de contenido presentó un acuerdo substantivo entre los jueces, con un índice de Lawshe de 0,822. La confiabilidad fue determinada con un alfa de Cronbach de 0,724.

Conclusiones: la versión en español del Florida Patient Acceptance Survey, es un instrumento fiable para medir la aceptación de las personas con implante de dispositivo de estimulación cardiaca.

(c) 2018 Sociedad Colombiana de Cardiología y Cirugía Cardiovascular. Publicado por Elsevier España, S.L.U. Este es un artículo Open Access bajo la licencia CC BY-NC-ND (http:// creativecommons.org/licenses/by-nc-nd/4.0/).
\end{abstract}

\footnotetext{
* Autor para correspondencia.

Correo electrónico: dmcastillos@unal.edu.co (D.M. Castillo-Sierra).
} 


\section{KEYWORDS}

Electrical

stimulation;

Device;

Validation studies;

Health care

acceptance;

Arrhythmias

\section{Validity and reliability of the Spanish version of the Florida Patient Acceptance Survey}

\begin{abstract}
Background: For the current health services, the measurement and generation of indicators that enable health outcomes to be evaluated, is of great relevance as part of quality health care. As a result, the adaptation and validation processes of measurement tools are important to ensure that they are reliable to use. The Florida Patient Acceptance Survey is a specific scale for measuring the acceptance of cardiac devices by individuals.

Method: A methodological-type study was carried out, in which the face validity, content and reliability of the Spanish version of the Florida Patient Acceptance Survey. The face validity and content was performed by expert consensus, using the statistical tests, Fleiss Kappa Coefficient and the modified Lawshe index. The theory of the item was used to perform the reliability test, in which a total of 126 individuals implanted with a cardiac stimulation device took part. The reliability was calculated using the Cronbach alpha statistical test.

Results: The face validity evaluates three categories: clarity, precision, and comprehension, showing a moderate agreement between the experts for the categories. There was a substantial agreement in content validity among the experts, with a Lawshe index of 0.822 . The reliability was determined with a Cronbach alpha of 0.724 .

Conclusions: The Spanish version of the Florida Patient Acceptance Survey, is a reliable tool to measure the acceptance of individuals with cardiac stimulation device implant.

(c) 2018 Sociedad Colombiana de Cardiología y Cirugía Cardiovascular. Published by Elsevier España, S.L.U. This is an open access article under the CC BY-NC-ND license (http:// creativecommons.org/licenses/by-nc-nd/4.0/).
\end{abstract}

\section{Introducción}

Los avances tecnológicos en el campo de la medicina, han permitido a las personas obtener nuevas formas de tratamiento para alteraciones cardiacas que ponen en riesgo la vida. Desde 1930, cuando apareció el primer marcapaso portátil, la estimulación cardiaca cobró importancia para mejorar la sobrevida de las personas, haciendo que su uso sea más frecuente y tenga un costo-beneficio relevante para los sistemas de salud, en términos de años de vida saludable con calidad de vida -AVAC-1-3. Los dispositivos de estimulación cardiaca tienen tanto funciones diagnósticas como terapéuticas y una gran complejidad técnica. A pesar de su evolución tecnológica a través del tiempo, aún las personas implantadas con este tipo de dispositivos, persisten con dificultades para la aceptación de los mismos en la vida cotidiana, ya que suponen algunas limitaciones y cambios en el estilo de vida ${ }^{2,4-10}$.

La aceptación como constructo abarca no solo cuestiones de carácter físico, sino también psicológico y social ${ }^{11-14}$. Constituye una herramienta fundamental para obtener el máximo de los beneficios que ofrece la tecnología. Por tanto, resulta relevante para el profesional de la salud evaluar todos los aspectos que influyen en las condiciones de salud de los individuos en aras de optimizar la calidad de la atención y obtener mejores resultados en términos de bienestar y salud de las personas implantadas. En la literatura actual, son escasos los instrumentos de medición específicos para las personas con dispositivos de estimulación cardiaca. No obstante, se encontró que el instrumento Florida Patient Acceptance Survey (FPAS, su sigla en inglés), es una medida específica para este grupo poblacional y en virtud de sus propiedades psicométricas óptimas, es un instrumento fiable para medir la aceptación de las personas a los dispositivos de estimulación cardiaca de una manera objetiva, definida esta como el alojamiento psicológico y la comprensión de las ventajas y desventajas del dispositivo, la recomendación del dispositivo a los demás y la obtención de beneficios en términos de funcionamiento biomédico, psicológico y social ${ }^{11}$.

El FPAS consta de 18 ítems con escala de respuesta tipo Likert. Evalúa cuatro categorías o dimensiones: retorno a la vida, distress relacionado con el dispositivo, valoración positiva y preocupaciones de la imagen corporal. La escala fue desarrollada a través de un estudio psicométrico en el 2005, cuando se reportó un alfa de Cronbach de 0,83 para toda la escala, mientras que para cada una de las categorías o dimensiones un coeficiente de Cronbach entre 0,74 a 0,89; así mismo, se demostró validez convergente con la escala de calidad de vida SF - 36 y validez divergente con las escalas CES - D y STAI ${ }^{11}$. De igual forma, la escala fue adaptada y validada en población danesa, en la que reportan que la validez del instrumento fue confirmada con los cuatro factores con un alfa de Cronbach que va de 0,73 a 0,85 , por lo que demostró ser una medida válida y fiable de aceptación al dispositivo en los pacientes que requieren estimulación cardiaca (tabla 1) ${ }^{15,16}$.

Este instrumento de origen anglosajón no cuenta con una versión en español; por tanto, el objetivo de este estudio es obtener la versión en idioma español adaptada al contexto colombiano, que conserve la estructura del instrumento original con propiedades psicométricas adecuadas para su implementación en la atención en salud de las personas con implante de dispositivos cardiacos. 
Tabla 1 Validez y fiabilidad del Florida Patient Acceptance Survey (FPAS)

\begin{tabular}{|c|c|c|}
\hline Prueba & Resultado & Población \\
\hline Validez convergente & $\begin{array}{l}\text { Correlación en ocho subescalas del SF - 36: Función } \\
\text { física, rol físico, dolor corporal, salud general, vitalidad, } \\
\text { social, rol emocional, salud mental. }\end{array}$ & Estadounidense \\
\hline Validez divergente & $\begin{array}{l}\text { No muestra correlación con los instrumentos STAI de } \\
\text { ansiedad y CES-D de síntomas depresivos. }\end{array}$ & Estadounidense \\
\hline Validez discriminante & $\begin{array}{l}\text { Habilidad para detectar diferencias entre grupos de } \\
\text { dispositivos. }\end{array}$ & Estadounidense \\
\hline Análisis factorial & Cuatro factores que explican el $42 \%$ de la varianza. & Estadounidense \\
\hline Consistencia interna & Alfa de Cronbach 0,83 para 15 ítems. & Estadounidense \\
\hline Consistencia interna & Alfa de Cronbach 0,85 para 15 ítems. & Danesa \\
\hline Análisis factorial & $\begin{array}{l}\text { Confirma los cuatro factores que explican el } 64,3 \% \text { de la } \\
\text { varianza }\end{array}$ & Danesa \\
\hline
\end{tabular}

Fuente: Castillo Sierra DM, González Consuegra RV. Intervención de enfermería para la aceptación del paciente al cardiodesfibrilador, aplicada en el preimplante.

\section{Materiales y métodos}

Es un estudio de tipo metodológico que aplica pruebas psicométricas para llevar a cabo los procesos de validez facial, validez de contenido, confiabilidad y consistencia interna, en este caso del instrumento "FPAS versión en Español". La psicometría procura fortalecer y dar evidencias empíricas de una u otra propiedad, conforme al propósito de la medición; garantiza que los instrumentos de medida sean debidamente estandarizados y posean valide $z^{17}$.

Las dos características métricas esenciales para valorar la precisión de un instrumento son la validez y la fiabilidad; la validez de una medida entendida como el grado en que proporciona información del fenómeno que pretende medir y no de otros ${ }^{18}$. Burns y Grove ${ }^{19}$ refieren que la validez de un instrumento es la determinación del alcance con que este es capaz de reflejar las características del concepto abstracto que se examina, mientras que para Polit y Hungler ${ }^{20}$ la validez constituye el segundo criterio de importancia para evaluar la idoneidad de un instrumento cuantitativo; denota el grado en que el instrumento mide lo que se supone que debe medir. En este caso, la validez facial o aparente es el grado en que una situación de medida parece ser válida desde el punto de vista del observador ${ }^{18}$ y la validez de contenido que se mide para evitar conclusiones inválidas a partir de una escala o instrumento. En esta está la información que se recoge de los ítems debe satisfacer adecuadamente los criterios conceptuales y el alcance de los mismos para explicar un fenómeno en salud ${ }^{18}$. Esta medición se logra a partir de criterios de un panel de expertos, quienes evalúan las variables con las que se define ${ }^{21}$.

Por otra parte, la fiabilidad se refiere a la exactitud con la que el instrumento mide el atributo para el cual está diseñado. Si los puntos que componen una escala miden, en teoría, el mismo constructo, los cuales deben mostrar una alta correlación, la escala debe mostrar un alto grado de homogeneidad ${ }^{22}$. Cronbach ha expresado que la confiabilidad de un instrumento de medición, se refiere al grado en que su aplicación repetida al mismo objeto o sujeto, produce iguales resultados, es decir a la condición de ser reproductible, en otras palabras la capacidad de mostrar resultados análogos en situaciones similares ${ }^{23}$.

\section{Procedimiento}

\section{Validez facial}

La validez facial se llevó a cabo mediante el juicio de expertos: una enfermera experta en dispositivos de estimulación cardiaca, dos enfermeras con experiencia en validación de instrumentos de medición y manejo de pacientes con alteraciones del rimo cardiaco, un enfermero psicólogo especialista en Salud Mental y un médico electrofisiólogo. Cada experto evalúo los criterios de claridad, precisión y comprensión de cada uno de los ítems, asignando el puntaje correspondiente (tabla 2).

\section{Validez de contenido}

Se realizó, así mismo, a partir del juicio de expertos. El objetivo del juicio consiste en evaluar qué tan relevantes y representativos son los ítems del instrumento para la aceptación del paciente al dispositivo. Para evaluar la validez de contenido se ha decido trabajar con los criterios de Lawshe modificados por Tristán, en tres categorías: esencial, útil pero no esencial y no necesario ${ }^{24}$.

\section{Confiabilidad}

Se hizo a través de la teoría del ítem, para lo cual se tomaron siete personas por ítem teniendo en cuenta que el instrumento FPAS versión en español tiene 18 ítems. En total participaron 126 personas con implante de dispositivos de estimulación cardiaca: marcapasos, cardiodesfibrilador y cardiorresincronizadores, para llevar a cabo la prueba de confiabilidad, previo diligenciamiento del consentimiento informado. 
Tabla 2 Criterios para evaluar la validez facial o aparente

\begin{tabular}{|c|c|c|c|}
\hline & & Puntaje & Observación \\
\hline $\begin{array}{l}\text { Claridad: se refiere a } \\
\text { que el ítem está }\end{array}$ & 1 & $\begin{array}{l}\text { Se otorga este valor cuando el ítem } \\
\text { no cumple con el criterio. }\end{array}$ & El ítem no es claro. \\
\hline \multirow[t]{2}{*}{$\begin{array}{l}\text { redactado de tal forma } \\
\text { que permite su fácil y } \\
\text { rápida comprensión. }\end{array}$} & 2 & $\begin{array}{l}\text { Se otorga este valor cuando el ítem } \\
\text { cumple moderadamente con el } \\
\text { criterio. }\end{array}$ & $\begin{array}{l}\text { El ítem requiere una modificación } \\
\text { específica en relación con el orden de } \\
\text { las palabras. }\end{array}$ \\
\hline & 3 & $\begin{array}{l}\text { Se otorga este valor cuando el ítem } \\
\text { cumple con el criterio. }\end{array}$ & $\begin{array}{l}\text { El ítem es claro, no requiere } \\
\text { modificación. }\end{array}$ \\
\hline $\begin{array}{l}\text { Precisión: se refiere a } \\
\text { que el ítem está }\end{array}$ & 1 & $\begin{array}{l}\text { Se otorga este valor cuando el ítem } \\
\text { no cumple con el criterio. }\end{array}$ & El ítem no es preciso. \\
\hline \multirow[t]{2}{*}{$\begin{array}{l}\text { redactado de forma } \\
\text { puntual y exacta; no da } \\
\text { lugar a dudas. }\end{array}$} & 2 & $\begin{array}{l}\text { Se otorga este valor cuando el ítem } \\
\text { cumple moderadamente con el } \\
\text { criterio. }\end{array}$ & $\begin{array}{l}\text { El ítem requiere una modificación } \\
\text { específica en relación con las } \\
\text { palabras para mejorar su exactitud. }\end{array}$ \\
\hline & 3 & $\begin{array}{l}\text { Se otorga este valor cuando el ítem } \\
\text { cumple con el criterio. }\end{array}$ & El ítem es preciso \\
\hline \multirow{3}{*}{$\begin{array}{l}\text { Comprensión: se refiere } \\
\text { a que al leer el ítem se } \\
\text { entiende lo que se } \\
\text { quiere decir. }\end{array}$} & 1 & $\begin{array}{l}\text { Se otorga este valor cuando el ítem } \\
\text { no cumple con el criterio. }\end{array}$ & El ítem no es comprensible. \\
\hline & 2 & $\begin{array}{l}\text { Se otorga este valor cuando el ítem } \\
\text { cumple moderadamente con el } \\
\text { criterio. }\end{array}$ & $\begin{array}{l}\text { El ítem requiere de una modificación } \\
\text { específica para poder interpretar su } \\
\text { significado. }\end{array}$ \\
\hline & 3 & $\begin{array}{l}\text { Se otorga este valor cuando el ítem } \\
\text { cumple con el criterio. }\end{array}$ & $\begin{array}{l}\text { El ítem es comprensible; permite } \\
\text { interpretar y entender su significado. }\end{array}$ \\
\hline
\end{tabular}

Fuente: Castillo Sierra DM, González Consuegra RV. Intervención de enfermería para la aceptación del paciente al cardiodesfibrilador, aplicada en el preimplante.

Tabla 3 Criterios George y Mallery

\begin{tabular}{ll}
\hline Coeficiente alfa $>0,9$ & Excelente \\
Coeficiente alfa $>0,8$ & Bueno \\
Coeficiente alfa $>0,7$ & Aceptable \\
Coeficiente alfa $>0,6$ & Cuestionable \\
Coeficiente alfa $>0,5$ & Inaceptable
\end{tabular}

Fuente: Gliem JA, Gliem RR. Calculating, interpreting, and reporting Cronbach's alpha reliability coefficient for Likert-type scales. Midwest Research-to-Practice Conference in Adult, Continuing, and Community Education.

\section{Consistencia interna}

La medida de la fiabilidad se efectuó mediante el coeficiente de alfa de Cronbach de acuerdo con el criterio general de George y Mallery, el cual asume que los ítems medidos en escala tipo Likert miden un mismo constructo y están altamente correlacionados ${ }^{25}$ (tabla 3)

\section{Análisis estadísticos}

Para las variables sociodemográficas se realizó un análisis descriptivo, utilizando distribución de frecuencias y porcentajes para realizar la caracterización de la muestra. Para la validez facial o aparente y la validez de contenido, se utilizaron las pruebas estadísticas del coeficiente de kappa de Fleiss, cuyo resultado se evalúa con la interpretación dada por Landis y Koch (tabla 4) y el índice de Lawshe modificado por Tristan, quien señala que se considera como aceptable cuando reporta un CVR' de 0.5823, independiente del número de expertos que se incluyen (72); de esta manera se
Tabla 4 Interpretación de Landis y Koch

\begin{tabular}{ll}
\hline $\mathrm{K}$ & Interpretación \\
\hline$<0$ & Poor agreement \\
$0,01-0,20$ & Slight agreement \\
$0,21-0,40$ & Fair agreement \\
$0,41-0,60$ & Moderate agreement \\
$0,61-0,80$ & Substantial agreement \\
$0,81-1,00$ & Almost perfect agreement
\end{tabular}

Fuente: Landis JR, Koch GG. The measurement of observer agreement for categorical data. Biometrics. 1977;33:159-74.

estableció la concordancia entre los jueces. La fiabilidad fue calculada a través de la prueba estadística del coeficiente de alfa de Cronbach.

\section{Resultados}

\section{Variables sociodemográficas}

La media de edad de los participantes fue de 68,37 DS \pm 14 años (fig. 1); 58,9\% pertenecía el género masculino frente a $41,1 \%$ del femenino. El nivel educativo fue bajo: el $70,2 \%$ correspondió a primaria y el $13,7 \%$ sin escolaridad. El $51,6 \%$ vivía en el área urbana y el $48,4 \%$ en área rural. $\mathrm{El}$ $66,9 \%$ pertenece al estrato socioeconómico 1 . El 35,5\% eran de estado civil casado, seguido del $24,2 \%$ viudez y $19,4 \%$ en unión libre. El 33,9\% estaba desempleado, el 26,6\% eran trabajadores independientes y el 36,6\% trabajaba en el hogar y eran mujeres. En relación con el tipo de dispositivo de estimulación cardiaca, el $58,9 \%$ de los participantes tenía 


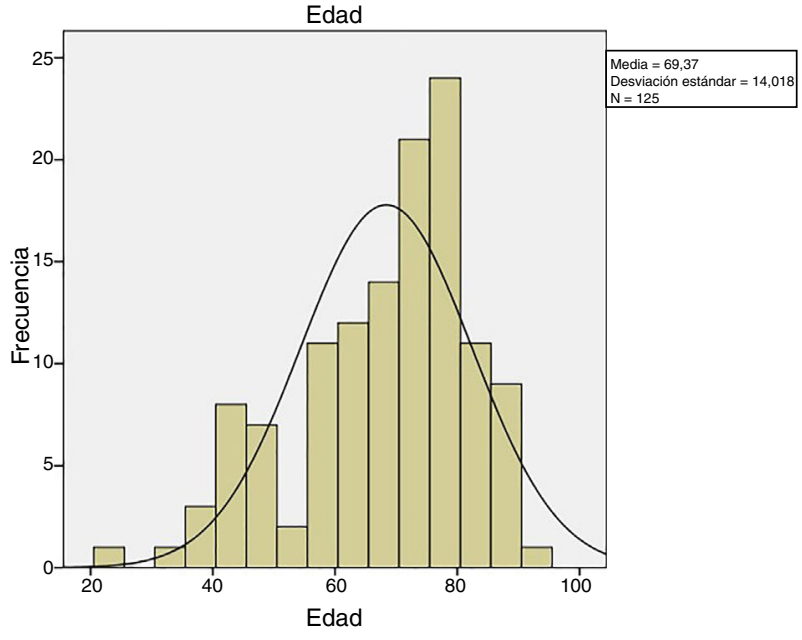

Figura 1

marcapasos, el 32,3\% cardiodesfibrilador y el 8,9\% cardiorresincronizadores. La media de tiempo del dispositivo con fue de 4,06 DS $\pm 4,5$ años de implantación. El 83,9\% refirió conocer la indicación médica del dispositivo y 16,1\% manifestó no conocerla. En la tabla 5 se aprecian los resultados en todas las variables sociodemográficas

\section{Validez facial}

Para determinar el grado de acuerdo entre los evaluadores respecto a la claridad, precisión y comprensión de cada uno de los ítems del instrumento, se calculó el índice de concordancia de kappa de Fleiss y el índice de Lawshe modificado. Los resultados muestran un acuerdo moderado entre los jueces para las categorías de claridad, precisión y comprensión (tabla 6).

\section{Validez de contenido}

Se evaluó a través de los expertos. Para tal fin se calculó el índice de Lawshe modificado y el índice de concordancia kappa de Fleiss. Para determinar si hubo un consenso entre los expertos se consideró un CVR' mayor 0,58; el resultado de la prueba estadística arrojo un índice de Lawshe modificado de 0,822 , que de acuerdo con la interpretación dada por Tristan es satisfactorio. En relación con el coeficiente kappa de Fleiss, el resultado 0,624, mostró un acuerdo substancial entre los jueces. Por tanto, los ítems evaluados representan el contenido de lo que la escala pretende evaluar, que es la aceptación al dispositivo de estimulación cardiaca (tabla 7).

\section{Confiabilidad}

El análisis estadístico se llevó a cabo mediante el uso del programa estadístico SPSS versión 24. La prueba del coeficiente de Cronbach de toda la escala es de 0,724 , por lo que el instrumento FPAS versión en español, es fiable para medir la aceptación del paciente al dispositivo de estimulación cardiaca.
Tabla 5 Variables sociodemográficas

\begin{tabular}{|c|c|}
\hline Género & \\
\hline Masculino & $58,9 \%$ \\
\hline Femenino & $41,1 \%$ \\
\hline Nivel educativo & \\
\hline Primaria & $70,2 \%$ \\
\hline Bachillerato & $11,3 \%$ \\
\hline Técnico & $0,8 \%$ \\
\hline Universitario & $3,2 \%$ \\
\hline Posgrado & $0,8 \%$ \\
\hline Ninguno & $13,7 \%$ \\
\hline Ubicación & \\
\hline Urbana & $51,6 \%$ \\
\hline Rural & $48,4 \%$ \\
\hline Estrato socioeconómico & \\
\hline Nivel 1 & $66,9 \%$ \\
\hline Nivel 2 & $27,9 \%$ \\
\hline Nivel 3 & $4,8 \%$ \\
\hline Nivel 4 & $0,8 \%$ \\
\hline Estado civil & \\
\hline Soltero & $15,3 \%$ \\
\hline Casado & $35,5 \%$ \\
\hline Unión libre & $19,4 \%$ \\
\hline Separado & $5,6 \%$ \\
\hline Viudo & $24,2 \%$ \\
\hline Ocupación & \\
\hline Empleado & $1,6 \%$ \\
\hline Independiente & $26,6 \%$ \\
\hline Desempleado & $33,9 \%$ \\
\hline Pensionado & $1,6 \%$ \\
\hline Otro & $36,3 \%$ \\
\hline Tipo de dispositivo & \\
\hline Marcapasos & $58,9 \%$ \\
\hline Cardiodesfibrilador & $32,3 \%$ \\
\hline Cardiorresincronizador & $8,9 \%$ \\
\hline
\end{tabular}

Tiempo del Dispositivo (años) Con una media de 4 +- DS 4,5 Fuente: Castillo Sierra DM, González Consuegra RV. Intervención de enfermería para la aceptación del paciente al cardiodesfibrilador aplicada en el preimplante.

Tabla 6 Validez facial

\begin{tabular}{lll}
\hline Criterio evaluado & Kappa de Fleiss & Lawshe modificado \\
\hline Claridad & 0,54 & 0,733 \\
Precisión & 0,42 & 0,678 \\
Comprensión & 0,60 & 0,79 \\
\hline
\end{tabular}

Fuente: Castillo Sierra DM, González Consuegra RV. Intervención de enfermería para la aceptación del paciente al cardiodesfibrilador aplicada en el preimplante.

\section{Discusión}

Los resultados de las variables sociodemográficas permitieron observar algunas tendencias propias del contexto colombiano, en las personas con un dispositivo de estimulación cardiaca. Una de ellas está relacionada con el 
Tabla 7 Estadísticas de fiabilidad

\begin{tabular}{lll}
\hline $\begin{array}{l}\text { Alfa de } \\
\text { Cronbach }\end{array}$ & $\begin{array}{l}\text { Alfa de Cronbach basado en } \\
\text { elementos estandarizados }\end{array}$ & $\begin{array}{l}\text { Número de } \\
\text { elementos }\end{array}$ \\
\hline 0,724 & 0,702 & 18 \\
\hline
\end{tabular}

Fuente: Castillo Sierra DM, González Consuegra RV. Intervención de enfermería para la aceptación del paciente al cardiodesfibrilador aplicada en el preimplante.

género, ya que la muestra difiere de otros estudios realizados en este grupo poblacional, en donde predomina el género masculino ${ }^{26-29}$. Los resultados muestran que la población femenina participante tiene un número importante de representación $(\mathrm{H}: 58,3 \%$ vs. $M: 41,1 \%)$, dato que puede llegar a ser importante para establecer diferencias por género frente a la experiencia de vivir con un dispositivo de estimulación cardiaca. Otro aspecto relevante tiene que ver con la ocupación de la población implantada, pues se encontró que la mayoría no tiene un empleo o trabaja como independiente, situaciones que afectan el rol social que desempeñan y la calidad de vida de las familias en las que se encuentran inmersos. De igual forma, el $48,4 \%$ de la población vive en área rural, otra característica crucial para la atención en salud de las personas implantadas, si se tiene en cuenta que el acceso a los servicios de salud en las zonas rurales colombianas tiene limitaciones significativas, frente a la oportunidad y el nivel de atención requerido para las personas con dispositivos cardiacos ${ }^{30,31}$

Por otra parte, la versión en español del instrumento FPAS, en la fase de adaptación transcultural para el contexto colombiano, tuvo modificaciones, las cuales no afectaron la estructura del instrumento original y lo que se pretende medir, hecho que fue corroborado a través de las pruebas de validez y fiabilidad, en las que se obtuvieron resultados aceptables, que son similares a la validación del instrumento original y a la versión adaptada en la población danesa ${ }^{11,15,16}$. De ahí que la escala permita medir la aceptación al dispositivo de las personas implantadas. Frente a las características sociodemográficas de los participantes, existen algunos aspectos similares, tales como la edad promedio que se encuentra por encima de los 60 años, en su mayoría población masculina, casados; al igual que el estudio de Burns et al. ${ }^{11}$ este estudio incluyó tres tipos de dispositivos cardiacos, sin embargo, la mayoría de los participantes de esta validación contaba con marcapasos y una proporción menor con otros dos tipos de dispositivos, hecho que difiere del estudio original del instrumento en cuanto a una distribución homogénea de la muestra frente al tipo de dispositivo.

La evaluación por parte de expertos en el área de las alteraciones del ritmo cardiaco y la salud mental, permite abordar de manera más homogénea el cuidado de enfermería y las necesidades de salud que tienen las personas con implante de un dispositivo de estimulación cardiaca en la vida cotidiana. Sin embargo, una de las dificultades de este estudio consistió en encontrar expertos, dado que se encuentran profesionales de enfermería con conocimientos prácticos en el área de Electrofisiología, pero con poco abordaje en la validación de instrumentos. De igual forma, este proceso de validación evidenció la importancia de abordar la aceptación de las personas al dispositivo, más allá de una mirada biotecnológica solamente, sino también de las necesidades psicológicas y sociales que influyen en su calidad de vida y en la manera de aprovechar los avances tecnológicos en la vida cotidiana.

Por consiguiente, cada vez es más necesario disponer de instrumentos de medida en el ámbito de la salud que se puedan utilizar en la práctica clínica e investigación ${ }^{32}$. La validación del instrumento permite tener una herramienta disponible para la práctica de enfermería en el cuidado de personas con dispositivos de estimulación cardiaca, de fácil acceso y aplicación, identificando necesidades de cuidado, así como estrategias específicas que puedan dar respuesta para mejorar el bienestar y la salud de las personas implantadas. Es importante señalar que una vez analizados los datos, se evidenció que la mayoría de los participantes a quienes se les aplicó el instrumento, pertenece a un estrato socioeconómico y nivel educativo bajo, circunstancia que fortalece la comprensibilidad del instrumento.

\section{Conclusión}

El Cuestionario de Florida para la aceptación del paciente, FPAS, en su versión en español, es un instrumento específico, válido y fiable para medir la aceptación al dispositivo de estimulación cardiaca de las personas implantadas en el contexto colombiano. Por tanto, esta herramienta puede ser utilizada en el ámbito clínico y en futuras investigaciones que aborden el cuidado y las necesidades de atención en salud de este grupo poblacional.

\section{Financiación}

Ninguna.

\section{Conflicto de intereses}

Ninguno.

\section{Bibliografía}

1. Alcaraz A, González-Zuelgaray J, Augustovski F. Costoefectividad del cardiodesfibrilador implantable en pacientes con factores de riesgo de muerte súbita en Argentina. Value Heal [Internet]. 2011;14:S33-8. Disponible en: http:// linkinghub.elsevier.com/retrieve/pii/S109830151101446X

2. Kirkfeldt RE, Johansen JB, Nohr EA, Jorgensen OD, Nielsen JC. Complications after cardiac implantable electronic device implantations: An analysis of a complete, nation wide cohort in Denmark. Eur Heart J. [Internet]. 2014;35. Disponible en: https://academic.oup.com/eurheartj/article-lookup/doi/10. 1093/eurheartj/eht511

3. Joyce KE, Lord S, Matlock DD, MCComb JM, Thomson R. Incorporating the patient perspective: a critical review of clinical practice guidelines for implantable cardioverter defibrillator therapy. J Interv Card Electrophysiol [Internet]. 2013;36:185-97. Disponible en: http://www.ncbi.nlm.nih.gov/pubmed/23250540

4. Palacios-Ceña D, Losa-Iglesias ME, Álvarez-López C, CachónPérez M, Reyes RAR, Salvadores-Fuentes P, et al. Patients, intimate partners and family experiences of implantable cardioverter defibrillators: Qualitative systematic review. 
J Adv Nurs. [Internet]. 2011;67:2537-50. Disponible en: http://doi.wiley.com/10.1111/j.1365-2648.2011.05694.x.

5. Palacios-Ceña D, Losa ME, Fernández-de-las-Peñas C, Salvadores-Fuentes P. Living with life insurance: A qualitative analysis of the experience of male implantable defibrillator recipients in Spain. J Clin Nurs. [Internet]. 2011;20(13-14), 2003-13 Disponible en: http://doi.wiley.com/10.1111/j.1365-2702.2010.03508.x.

6. Westerdahl AK, Sjöblom J, Mattiasson AC, Rosenqvist M, Frykman V. Implantable cardioverter-defibrillator therapy before death: High risk for painful shocks at end of life. Circulation. [Internet]. 2014;129(4.). Disponible en: http://circ.ahajour nals.org/cgi/doi/10.1161/CIRCULATIONAHA.113.002648

7. Shalaby A, Brumberg G, El-Saed A, Saba S. Mood disorders and outcome in patients receiving cardiac resynchronization therapy. Pacing Clin Electrophysiol [Internet]. 2012;35:294-301, http://dx.doi.org/10.1111/j.1540-8159.2011.03304.x.

8. Dunbar SB, Dougherty CM, Sears SF, Carroll DL, Goldstein NE, Mark DB, et al. Educational and psychological interventions to improve outcomes for recipients of implantable cardioverter defibrillators and their families: A scientific statement from the american heart association. Circulation [Internet]. 2012;126:2146-72. Disponible en: http://doi.wiley.com/10.1161/CIR.0b013e3591825fd

9. Habibović M, Burg MM, Pedersen SS. Behavioral interventions in patients with an implantable cardioverter defibrillator: lessons learned and where to go from here? Pacing Clin Electrophysiol [Internet]. 2013;36:578-90. Disponible en: http://www. pubmedcentral.nih.gov/articlerender.fcgi?artid=3809759\&tool =pmcentrez\&rendertype=Abstract

10. Saito NAO, Taru C, Miyawaki I, Illness Experience:. Living with Arrhythmia and Implantable Cardioverter Defibrillator. Kobe J. Med. Sci [Internet]. 2012;58:72-81.

11. Burns JL, Serber ER, Keim S, Sears SF. Measuring Patient Acceptance of Implantable Cardiac Device Therapy: J Cardiovasc Electrophysiol [Internet]. 2005;16:384-90. Disponible en: http://doi.wiley.com/10.1046/j.1540-8167.2005.40134.x

12. Pedersen SS, Spindler H, Johansen JB, Mortensen PT. Clustering of poor device acceptance and Type D personality is associated with increased distress in Danish cardioverter-defibrillator patients. Pacing Clin Electrophysiol [Internet]. 2009;32:29-36. Disponible en: http://www.ncbi.nlm.nih.gov/pubmed/19140910

13. Luderitz B, Jung W, Deister A, Manz M. Patient acceptance of implantable cardioverter defibrillator debvices: Changing attitudes. Am Heart J [Internet]. 1994;127:1179-84, http://dx. doi.org/10.1016/0002-8703(94)90107-4\&atitle=Patient+a.

14. Burke LJ. Securing life through technology acceptance: The first six months after transvenous internal cardioverter defibrillator implantation. Hear Lung J Acute Crit Care [Internet]. 1996;25:352-66. Disponible en: http://www.scopus.com/ inward / record.url?eid=2-s2.0-0029983943\&partnerID=40\&md5

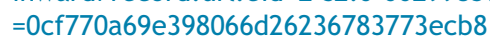

15. Versteeg $H$, Starrenburg A, Denollet J, Palen JVD, Sears SF, Pedersen SS. Monitoring Device Acceptance in Implantable Cardioverter Defibrillator Patients Using the Florida Patient Acceptance Survey. Pacing Clin Electrophysiol [Internet]. 2012;35:283-93. Disponible en: http://doi.wiley.com/10.1111/j.1540-8159.2011.03299.x

16. Pedersen SS, Spindler H, Johansen JB, Mortensen PT, Sears SF. Correlates of patient acceptance of the cardioverter defibrillator: Cross-validation of the Florida patient acceptance survey in Danish patients. Pacing Clin Electrophysiol
[Internet]. 2008;31:1168-77. Disponible en: http://doi.wiley. $\mathrm{com} / 10.1111 / \mathrm{j} .1540-8159.2008 .01158 . x$.

17. Rodríguez-Jiménez $O$, Rosero-Burbano R, Botia Sanabria $M$, Duarte Mateus L. Producción De Conocimiento En Psicometría En Instituciones De Educación Superior De Bogotá Y Chía. Rev Colomb Psicol [Internet]. 2011;20:9-25. Disponible en: http://revistas.unal.edu.co/index.php/psicologia/article/view $/ 16270 / 28671$

18. Ricardo S. Validación de Escalas de Medición en Salud. Rev Salud pública [Internet]. 2004;6:302-18.

19. Burns N, Grove SK. Investigación en enfermería+ Evolve: Desarrollo de la práctica enfermera basada en la evidencia. Elsevier; 2012.

20. Polit, D\& Hungler B. Investigación científica en ciencias de la salud. S. Edición. México; 2000.

21. Escobar-Pérez J, Cuervo-Martínez Á. Validez de contenido y juicio de expertos: una aproximación a su utilización. Av en Medición[Internet]. 2008;6:27-36.

22. Oviedo H. Propiedades psicométricas de una Escala: La Consistencia Interna. Rev Salud pública [Internet]. 2008;10:831-9.

23. Vesga Gualdrón Lucy Marcela, Ruiz de Cárdenas Carmen Helena. Validez y confiabilidad de una escala de cuidado profesional en español. av.enferm[Internet]. 2016;34:69-78, http://dx.doi.org/10.15446/av.enferm.v34n1.44488.

24. Tristan A. Modificación al modelo de Lawshe para el dictamen de validez de contenido de un instrumento objetivo. Av en medición [Internet]. 2008;6:37-48.

25. Oviedo Celina H, Campo-Arias A. Aproximación al uso Coeficiente Alfa de Cronbach. Rev Colomb Psquiatría [Internet]. 2005;34:572-80. Disponible en: http://www.scielo.org.co/pdf/rcp/v34n4/v34n4a09.pdf

26. Christensen AV, Zwisler AD, Svendsen JH, Pedersen PU, Blunk L, Thygesen LC, et al. Effect of cardiac rehabilitation in patients with ICD: Are gender differences present results from the CopeICD trial. Pacing Clin Electrophysiol [Internet]. 2015; 38(1). Disponible en: http://doi.wiley.com/10.1111/pace.12507

27. Van Den Broek KC, Heijmans N, Van Assen MaLM. Anxiety and depression in patients with an implantable cardioverter defibrillator and their partners: a longitudinal study. Pacing Clin Electrophysiol [Internet]. 2013;36:362-71. Disponible en: https://doi.org/10.1111/pace.12055

28. Cinar FI, Tosun N, Kose S. Evaluation of an education and follow-up programme for implantable cardioverter defibrillator-implanted patients. J Clin Nurs. [Internet]. 2013;22(17-18):2474-86. Disponible en: http://doi.wiley.com/10.1111/jocn.12201

29. Christensen AV, Zwisler AD, Svendsen JH, Pedersen PU, Blunk L, Thygesen LC, et al. Effect of cardiac rehabilitation in patients with ICD: Are gender differences present results from the COPE-ICD trial. PACE - Pacing Clin Electrophysiol. [Internet]. 2015;38(1.). Disponible en: http://doi.wiley.com/10.1111/pace.12507

30. Restrepo-Zea JH, Silva-Maya C, Andrade-Rivas F, VH-Dover R. Acceso a servicios de salud: análisis de barreras y estrategias en el caso de Medellín. Colombia. Gerenc y Políticas Salud [Internet]. 2014;13:242-65. Disponible en: http://revistas. javeriana.edu.co/index.php/gerepolsal/article/view/11970

31. ONS. Informe Tecnico Carga de Enfermedad por Enfermedades Crónicas no Transmisibles y Discapacidad en Colombia. 2015.

32. Carvajal A, Centeno C, Watson R, Martínez MS. ¿Cómo validar un instrumento de medida de la salud? An Sist Sanit Navar [Internet]. 2011;34:63-72. Disponible en: http://scielo.isciii. es /scielo.php?script=sci_arttext\&pid=S1137-6627201100010000 